\title{
LA COMUNICACIÓN DE LA VERDAD EN LA RELACIÓN MÉDICO-PACIENTE TERMINAL
}

\begin{abstract}
Alejandra Gajardo Ugás*
Resumen: Las personas que trabajan en cuidados paliativos se enfrentan a muchos dilemas éticos. A menudo, ellos se relacionan con el deber de decir la verdad y respetar la autonomía del paciente, en oposición al deber de cuidar y no provocar daño. El principio de autonomía se basa en el respeto a los valores, creencias y capacidad de tomar decisiones del paciente como la principal consideración moral y, por tanto, exige la comunicación de la verdad. La beneficencia, al buscar el predominio del bien en la asistencia del paciente y procurar su beneficio, supone que la verdad puede dañar.
\end{abstract}

Palabras clave: paciente terminal, comunicación, verdad

\section{COMMUNICATION OF TRUTH IN THE DOCTOR-TERMINAL PATIENT RELATIONSHIP}

Abstract: People who work at palliative care face many ethical dilemmas. Often they are related with the duty to tell the truth to the patient and to respect his autonomy, in opposition to the duty of caring and not harming.

The autonomy principle is based upon respect for the patient's values, beliefs and capacity of making decisions as the principal moral consideration and, therefore, it requires communication of the truth. Beneficence, when looking for the patient's sake and for his benefit, can suppose that truth may cause damage.

Key words: terminal patient, communication, truth

\section{A COMUNICAÇÃO DA VERDADE NA RELAÇÃO MÉDICO-PACIENTE TERMINAL}

Resumo: As pessoas que trabalham em cuidados paliativos enfrentam muitos dilemas éticos. Em geral, eles se relacionam com o dever de dizer a verdade e respeitar a autonomia do paciente, em oposiçáo ao dever de cuidar e náo provocar dano. O principio da autonomia se baseia no respeito a valores, crenças e na capacidade de tomar decisóes do paciente como a principal consideraçáo moral e que, portanto, exige a comunicação da verdade. A beneficência, ao buscar o predomínio do bem na assistência do paciente e a procura de seu beneficio, supóe que a verdade pode provocar dano.

Palavras-chave: paciente terminal, comunicação, verdade 


\section{Introducción}

Los profesionales del área de la salud se ven enfrentados frecuentemente a dilemas éticos en el cuidado de pacientes terminales. Algunos ejemplos corresponden a la decisión acerca de la conveniencia de dar a conocer al enfermo su diagnóstico o pronóstico(1). Decir o no la verdad a un paciente puede parecer muy simple, pero en realidad es una decisión muy difícil. La comunicación de la verdad, particularmente cuando se trata de informar "malas noticias", es una situación estresante y difícil para los médicos y para el resto del equipo de salud(2). La comprobación habitual es que esta comunicación es temperada de modo tal que, en el mejor de los casos, resulta en una verdad parcial, y en el peor en un cuadro pintado sobre la base de falsedades y omisiones.

La ética médica tradicional se ha basado en dos principios fundamentales, "beneficencia" y "no maleficencia", exigidos a los médicos en ejercicio(3). Estos principios se enfrentan frecuentemente cuando el médico debe entregar información que puede alterar en forma negativa la visión del paciente sobre su situación de salud.

\section{La comunicación de la verdad}

En un contexto clínico resultaría incorrecto por muchas razones mentir, pero reducir o postergar la revelación total de la verdad podría ser justificable moralmente. $\mathrm{Si}$ un paciente se encuentra emocionalmente afectado, deprimido y con actitudes suicidas, entonces se requiere de cautela para que la revelación de la verdad no contribuya a un severo perjuicio(4). En este caso, en vez de mentira se podría hablar de un ocultamiento de la verdad.

Este ocultamiento puede tomar diferentes formas, la mayor parte de ellas relacionadas con la forma de la entrevista en que la verdad debe ser comunicada; también puede tener muchos propósitos y llevar a diversas consecuencias. En general, la postura de ocultamiento ha sido sostenida bajo el principio de no maleficencia, aduciendo que, en algunos casos, la verdad puede causar un daño al paciente(5). Este manejo o manipulación de la verdad, que suele utilizarse en la comunicación del diagnóstico y pronóstico de enfermedades terminales, y que estaría avalando uno de los deberes de la profesión médica como es la beneficencia, es entendido como solidaridad con el enfermo que sufre, que padece un mal doloroso que afecta su salud y que amenaza su vida.
El atenuar la angustia o la depresión, productos de una verdad dolorosa, podría ser una motivación suficiente para ocultar la verdad.

El hecho de ocultar la verdad en la relación médicopaciente terminal requiere de especial atención, ya que en la actualidad los pacientes demandan más información sobre las opciones de tratamiento, riesgo, técnica quirúrgica, pronóstico, entre otros aspectos. Esto ha generado incluso un cambio en nuestras leyes. Los pacientes podrían experimentar un daño si se les miente. No sólo se pasaría a llevar su autonomía, sino que, además, aquellos pacientes que no están al tanto de la verdad acerca de las intervenciones experimentan una pérdida total de la confianza en el médico, la cual es fundamental para el proceso de cuidados.

\section{La relación médico-paciente}

El actual modelo de relación médico-paciente no ha logrado imponerse al modelo clásico paternalista, en el que una gran parte de la información referente al mal que padecía el paciente se mantenía inaccesible para él. En nuestro país el médico todavía asume, en muchos casos, la responsabilidad general en el tratamiento de los enfermos, a pesar de la obligación de aceptar que el paciente tiene derecho a ejercer su autonomía en la atención médica. Entre los médicos existe la percepción de una invasión en el ejercicio de la profesión, por el protagonismo que está alcanzando la autonomía de los pacientes, proceso en el cual el médico va perdiendo progresivamente el control sobre su práctica. La creciente participación de los pacientes también enfrenta a los profesionales a reconocer la incertidumbre y producir frustración en quienes creen en la certeza definitiva de la medicina(6). Esto, que podría ser una curiosidad histórica, sigue siendo importante hoy al persistir vivas las raíces de una relación paternalista, moduladora de la entrega de la verdad.

Por otro lado, son diferentes las valoraciones que los propios pacientes muestran acerca de la importancia del ejercicio de su autonomía en las decisiones de salud. Vemos usuarios del sistema privado de salud que defienden cada vez más sus derechos, mientras que otros se encuentran a gusto con el clásico modelo paternalista y les incomoda la libertad de opción que ofrece el médico(7).

Actualmente, el tipo de relación interpersonal entre médico y paciente ofrece características poco vincu- 
lantes y tecnologizadas. Ello se debe a las condiciones en las cuales se desarrolla la consulta, a la ausencia de un médico de cabecera y a una atención multidisciplinaria(8).

$\mathrm{Al}$ iniciar una relación de terapia o investigación, el paciente y el médico subscriben un contrato tácito, mediante el cual ambos se comprometen con el derecho y el deber de decir la verdad el uno al otro, una de las condiciones indispensables para que se establezca entre ellos una relación de confianza(9). Sin embargo, frente a una declaración que puede parecer clara y aceptada como ésta, decir la verdad sigue siendo uno de los dilemas éticos más relevantes en el acompañamiento de los pacientes terminales(10), existiendo quienes se preguntan si el médico es capaz realmente de saber la verdad y de pronosticarla, o si el paciente quiere saber la verdad o es capaz de entenderla.

En el caso de los enfermos terminales se presentan siempre conflictos entre el derecho a conocer la verdad, por una parte, en consideración con el principio de autonomía, y los principios ya citados de beneficencia y no maleficencia, por otra. En este conflicto juega un papel fundamental el proceso de comunicación(11).

\section{El derecho y el deber de comunicar la verdad}

Si bien el paciente terminal tiene derecho a conocer todo lo concerniente a su enfermedad y su proceso de morir, tiene también derecho a rehusar conocerlo, lo que suele traducirse en no indagar nada al respecto $y$, por lo tanto, el médico se sentiría sin el deber de informar. Esto podría generar uno de los elementos que avalarían la licitud del ocultamiento de la verdad.

Establecer una comunicación abierta con el paciente terminal es para los profesionales de la salud un asunto difícil de salvar en la práctica diaria. La muerte y el proceso de morir evocan en médicos y enfermeras reacciones psicológicas que conducen, directa o indirectamente, a evitar la comunicación sobre la materia con el paciente y su familia. Nuestra sociedad vive de espaldas a la muerte. Por otro lado, cuando las personas están muriendo, la etapa de negación de la muerte crea una barrera entre ellas y el resto de la sociedad, que las aísla cuando más ayuda necesitan.
La información entregada al paciente terminal deberá combinar la prudencia necesaria para no dańar las posibilidades de adaptación del enfermo a la verdad. Si bien las circunstancias sociales y culturales van cambiando, en las sociedades latinoamericanas el médico y la familia suelen tender a creer que el ideal es que el paciente muera sin conciencia de su situación.

Estudios en Espańa revelan la existencia en este medio de al menos dos tipos de enfermo. El primero lo integran aquellos que desean conocer su diagnóstico (quienes lo más probable es que sean jóvenes, de sexo masculino y tengan una supervivencia esperada corta), pero a los que no siempre se informa. De éstos, el 12\% de enfermos no informados desearía una revelación plena de su diagnóstico, cifra que en otros estudios oscila entre el $50 \%$ y el $98 \%$, dependiendo de las características demográficas y del diagnóstico sospechado por el enfermo(12).

\section{Conclusión}

Finalmente, cuando la comunicación se da en la relación médico-paciente terminal, el respeto, la veracidad, la confianza y la beneficencia cobran importancia, así como la individualidad del enfermo al que nos enfrentamos. En esta situación, la meta es la calidad de vida y no alargar la supervivencia ni producir daños innecesarios. Para ello hay que decidir los aspectos que van a ser abordados en el proceso de la información: diagnóstico, tratamiento, pronóstico y/o apoyo. Cambiar el miedo por esperanza, la incertidumbre por información, la indefensión por percepción de control, la incomunicación por una comunicación adecuada, abierta y honesta no es fácil. Las variaciones individuales son tantas y son ellas, y no las generalidades, las que nos deben guiar durante el proceso de comunicación(13).

El respeto y la confianza mutuos se hacen imprescindibles en el trato entre paciente y profesional. Sin embargo, en la actualidad este punto de partida no siempre se cumple, debido a una evolución sociocultural que supone mayor implicación del paciente en todos los procesos de salud; mayor exigencia de eficacia; mala o escasa información y gran número de profesionales que participan en un solo proceso terapéutico, lo que dificulta aún más la comunicación de la verdad a los pacientes. 


\section{Referencias}

1. Taboada P. Principios relevantes en la atención de pacientes terminales. Revista de Estudios Médico-Humanisticos; 12(12). Sitio en Internet. Disponible en http://escuela.med.puc.cl/publ/ArsMedica/ArsMedica12/PrincipiosEticos.html

2. Bascunán ML. Comunicación de la verdad en medicina: contribuciones desde una perspectiva psicológica. Revista Médica de Chile 2005; 133(6): 693-698.

3. Amaro C, et al. Principios básicos de la bioética. Revista Cubana de Enfermería 1996; 12(1): 11-12.

4. Simone G. El final de la vida: situaciones clínicas y cuestionamientos éticos. Acta Bioethica 2000; 6(1): 47-62.

5. Franca O, et al. La ayuda (médica) al buen morir. Ars Médica 2005; 11.

6. Bascunán ML. Cambios en la relación médico-paciente y nivel de satisfacción de los médicos. Revista Médica de Chile 2005; 133(1): 11-16.

7. León F. Autonomía y beneficencia en la ética clinica: ni paternalismo ni Medicina defensiva. Sitio en Internet. Disponible en https://www.u-cursos.cl/medicina/2007/2/KINEBIOET12/1/material_docente/objeto/146380

8. Lavados M, Serani A. Ética Clínica: Fundamentos y Aplicaciones. Santiago de Chile: Editorial Universidad Católica de Chile, Colección Textos Universitarios; 1993: 348.

9. Vacarezza R. De los derechos del paciente. Revista Médica de Chile 2000; 128(12): 1380-1384.

10. Twycross R. Medicina paliativa: filosofía y consideraciones éticas. Acta Bioethica 2000; 6(1): 29-46.

11. Astudillo W. ¿Cómo mejorar la comunicación en la fase terminal? Ars Médica 2005; 11: 61-85.

12. Centeno-Cortés $C$, Núnez Olarte JM. Cuestionar la revelación del diagnóstico a los enfermos oncológicos en situación terminal: un estudio prospectivo que evalúa las respuestas de los enfermos. Cuadernos de Bioética 1996; 7(26): 156163.

13. Astudillo W, Mendinueta C. Bases para mejorar la comunicación con el enfermo terminal. En: Astudillo W, (ed.) Cuidados del enfermo en fase terminal y atención a su familia. Navarra: EUNSA; 1995: 42-51.

Recibido: 18 de abril de 2008

Aceptado: 24 de junio de 2008 